\title{
Carrion fly-derived DNA metabarcoding is an effective tool for mammal surveys: evidence from a known tropical mammal community
}

Torrey W. Rodgers $* \mathbf{1 , 2}$, Charles C. Y. Xu $\mathbf{3}^{\mathbf{3} 4,5}$, Jacalyn Giacalone ${ }^{6}$, Karen M. Kapheim ${ }^{\mathbf{7}, 2}$, Kristin

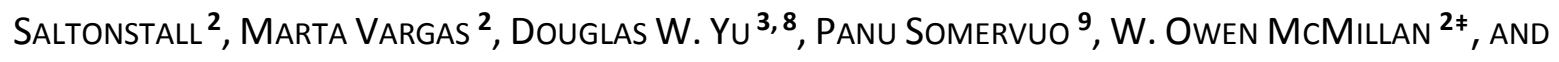
PATRICK A. JANSEN ${ }^{2,10 \neq}$

${ }^{1}$ Department of Wildland Resources, Utah State University, 5230 Old Main Hill, Logan, UT 84322, USA

${ }^{2}$ Smithsonian Tropical Research Institute, Apartado 0843-03092, Balboa, Panama

${ }^{3}$ State Key Laboratory of Genetic Resources and Evolution, Kunming Institute of Zoology, Chinese Academy of Sciences, Kunming, Yunnan 650203, China.

${ }^{4}$ Groningen Institute for Evolutionary Life Sciences, University of Groningen, P.O. Box 11103, 9700 CC Groningen, The Netherlands.

${ }^{5}$ Redpath Museum and Department of Biology, McGill University, Montreal, QC, Canada.

${ }^{6}$ College of Science and Mathematics, Montclair State University, Montclair, NJ 07043 USA

${ }^{7}$ Department of Biology, Utah State University, 5305 Old Main Hill, Logan, UT 84322, USA

${ }^{8}$ School of Biological Sciences, University of East Anglia, Norwich Research Park, Norwich, Norfolk NR4 7TJ, UK;

${ }^{9}$ Department of Biosciences, University of Helsinki, Finland

10 Department of Environmental Sciences, Wageningen University, PO Box 47, 6700 AA Wageningen, The Netherlands

¥ These authors jointly advised this work

* Corresponding author torrey.w.rodgers@gmail.com

1 Keywords: Barro Colorado Island, Biodiversity, camera trapping, eDNA, transect counts

2 Running title: Carrion fly metabarcoding for mammal surveys 
Metabarcoding of vertebrate DNA derived from carrion flies has been proposed for biodiversity monitoring. To evaluate its efficacy, we conducted metabarcoding surveys of carrion flies on Barro Colorado Island (BCl), Panama, which has a well-known mammal community, and compared our results against diurnal transects and camera-trapping. We collected 1084 flies in 29 sampling days and conducted metabarcoding with mammal-specific (16S) and vertebrate-specific (12S) primers. For taxonomic assignment, we compared BLAST with the new program PROTAX, and we found that PROTAX

10 improved species identifications. We detected 20 mammal, four bird, and one lizard species via fly 11 metabarcoding, all but one of which are known from $\mathrm{BCl}$. Twenty-nine days of fly metabarcoding

12 detected more mammal species than did concurrent transects (29 sampling days, 13 species) and

13 concurrent camera-trapping (84 sampling days, 17 species). Fly metabarcoding also detected $67 \%$ of the 14 mammal species documented by eight years of transects and camera-trapping combined. However, fly metabarcoding (almost entirely) missed four of BCl's most abundant mammal species, three of which

16 have pellet feces. This study demonstrates that fly metabarcoding indeed has the potential to accurately

17 detect a broad range of vertebrates but is probably best used as an efficient complement to cameratrapping and visual transects.

\section{Introduction}

20 Due to the rapid decline in biodiversity worldwide, there is an urgent need for more efficient

21 techniques to survey and monitor biodiversity. Surveys based on direct observation are costly and time

22 consuming, detection rates vary across observers, and rare or cryptic species are often overlooked.

23 Camera trapping has emerged as a more efficient survey method, especially for large-bodied

24 vertebrates (Beaudrot et al. 2016), but it has limited ability to detect small-bodied, arboreal, and volant 25 species. Moreover, camera traps are expensive for use at large scales and are subject to damage and 
theft. An efficient technique capable of detecting a wide range of vertebrate species would increase our

27 ability to monitor vertebrate diversity, and to gauge the effectiveness of conservation measures such as nature reserves.

One potential method has emerged from the field of environmental DNA (eDNA), which uses trace amounts of DNA for species detection (Bohmann et al. 2014). This approach shows great potential for efficient monitoring of biodiversity (Ji et al. 2013; Taberlet et al. 2012; Yu et al. 2012). To date, the majority of vertebrate eDNA research has focused on aquatic species because eDNA is easy to collect from water (Thomsen et al. 2012). Collecting eDNA from terrestrial vertebrates is more difficult. Researchers have tried soil (Andersen et al. 2012), browsed twigs (Nichols et al. 2012), prey carcasses (Wheat et al. 2016), and drinking water (Rodgers \& Mock 2015) for eDNA detection of terrestrial species, but these approaches have limited scope. So far, the most promising approach for use across a broad range of species is mass trapping and metabarcoding of invertebrates that feed on vertebrates (Bohmann et al. 2013; Calvignac-Spencer et al. 2013a). Such invertebrate 'samplers' tested to date include leeches (Schnell et al. 2015), mosquitoes (Logue et al. 2016), dung beetles (Gillett et al. 2016), and carrion flies (Calvignac-Spencer et al. 2013b; Ping Shin Lee et al. 2016; P. S. Lee et al. 2015; Schubert et al. 2014). We focus on the latter. upon dead animals, open wounds, and feces. When carrion flies feed, they ingest vertebrate DNA.

44 Carrion flies are ideal candidates for eDNA surveys because they are easy to trap, are ubiquitous worldwide, and are believed to feed opportunistically on vertebrates of all sizes, including terrestrial species, volant species, and species occupying the forest canopy. In a remarkable study, Calvignac-

47 Spencer et al. (2013b) used Sanger sequencing to detect 20 different mammal species from just 115 flies 
metabarcode a subset of samples, and were able to detect the majority of species that had been

50 detected with Sanger sequencing, plus several others.

51 Although carrion fly metabarcoding is promising, there is still a need to quantify its effectiveness

52 relative to conventional methods. There is also a need to test new methods for assigning taxonomies to

53 sequence data. This step may be particularly error prone in eDNA studies, because target amplicons for

54 eDNA are short due to the need to amplify degraded DNA, and thus have lower taxonomic information

55 content. Also, because reference sequence databases are incomplete, taxonomic assignment software

56 has a bias toward overconfidence, meaning that an operational taxonomic unit (OTU) sequence can be

57 assigned to a similar species that happens to be in the database when the correct species is not in the

58 database (Somervuo et al. 2017). Correct species-level identification is especially important when

59 applied to vertebrates, as incorrect assignment of an OTU to an endangered species, as opposed to a

60 less endangered congener (or vice versa), can have a large impact on conservation decision making.

61 With these goals in mind, we conducted a field test on Barro Colorado Island (BCl) in Panama,

62 where the vertebrate fauna is well documented, particularly for mammals. We collected carrion flies for

63 metabarcoding and compared results with datasets from annual, diurnal transect counts (distance

64 sampling) and semi-continuous camera-trapping of the mammal community for eight years leading up

65 to, and concurrent with, fly collection. In addition, we compared taxonomic assignments between the

66 most commonly used method, BLAST (Altschul et al. 1990) and the new method PROTAX (Somervuo et

67 al. 2017), which uniquely takes into account the possibility that an OTU sequence belongs to a species

68 that is not in the reference database, thus avoiding overconfident assignments. We find that

69 metabarcoding of carrion flies is an effective, but imperfect, method for surveying mammal

70 communities, and PROTAX outperforms BLAST for taxonomic assignment. 


\section{Materials and Methods}

\section{Study site}

73

74

75

76

77

78

79

80

81

82

83

84

85

86

87

88

89

90

91

92

Fieldwork was conducted on Barro Colorado Island, a 1,560-ha island in the Panama Canal waterway (Fig. 1). $\mathrm{BCl}\left(9^{\circ} 10^{\prime} \mathrm{N}, 79^{\circ} 51^{\prime} \mathrm{W}\right)$ sits within Gatun Lake, an artificial body of water created in 1912 by the damming of the Chagres River to create the Panama Canal, and is part of the protected $54-\mathrm{km}^{2}$ Barro Colorado Nature Monument. $\mathrm{BCl}$ has 108 known mammal species, including 74 bats and 34 non-volant species (Glanz 1982 and current expert information). However, some of these species such as jaguar (Panthera onca), puma (Puma concolor), and jaguarundi (Herpailurus yagouaroundi) are only infrequent visitors to the island.

\section{Fly collection}

Flies were collected between 10 Feb and 5 May 2015 in three trapping sessions totaling 620 fly-trap days. All flies collected were used in the metabarcoding analysis. First, flies were collected from 10-14 Feb using a variety of trap types within $1000 \mathrm{~m}$ of the labs on $\mathrm{BCl}$, to determine the best methods for sampling. Sampling methods tested included netting flies above covered bowls of pork and the trap types described in Calvignac-Spencer et al. (2013b). Based on results from this testing, we used a trap type modified from www.blowflies.net/collecting.htm for all subsequent trapping. Traps were baited with raw pork hung in a cup surrounded by fine cloth netting to keep flies from landing directly on the bait, and bait was replaced every 2-3 days. From 15-23 Feb, flies were collected from traps placed along trails at 16 trap locations, with one trap per location, spaced roughly 200 m apart in a non-uniform grid

(Fig 1). Second, from $28 \mathrm{Feb}-4$ Mar, traps were placed along trails at the same 16 trap locations as session 1, but with two traps per location. Third, from 12 Mar to 5 May 2015, flies were collected from traps placed in a transect crossing the island with 16 trap locations, each containing 2 traps, placed 
every $250 \mathrm{~m}$ along a trial in a roughly straight line (Fig 1), for 10 sampling days. In all three collection

94 efforts, flies were removed from traps once or twice daily and placed in a $-40^{\circ} \mathrm{C}$ freezer within 2 hours of collection, because DNA degradation causes detection success to decline 24 hours after fly feeding (P. S. Lee et al. 2015).

\section{Library preparation and sequencing}

DNA was extracted from flies using the GeneMATRIX Stool DNA Purification Kit (Roboklon, Berlin,

Germany). To reduce extraction costs, up to 16 flies were pooled for each extraction. Flies were first cut into several pieces with sterile scissors and placed into $2.5 \mathrm{ml}$ Polypropylene 96 Deep Well Plates along with stainless steel, 5/32" grinding balls (OPS diagnostics; Lebanon, NJ). Up to 4 flies and $100 \mu$ l of lysis buffer per fly were added to each well. Plates were shaken on a TissueLyser II (Qiagen; Germantown, MD) until fly tissue was homogenized in the lysis buffer. An equal volume of the resulting homogenate per fly was then pooled in a total volume of $160 \mu \mathrm{l}$, and added to the extraction kit bead tube. Extractions then proceeded following manufacturer's recommendations. For flies collected in the first trapping session, each extraction included 16 flies. For flies collected in the second and third trapping sessions, each extraction contained all flies collected at the same trap location on the same day, up to a maximum of 16 flies. To examine if pooling had an effect on species detection, 24 flies were also homogenized and extracted individually, and $20 \mu \mathrm{l}$ of homogenate from each of these same 24 flies was also pooled and extracted in 2 samples containing 12 flies each. Single-fly and pooled samples were all

111 sequenced at the same depth. All extractions included blank controls that were included in the PCR step 112 to test for contamination.

114 Boessenkool et al. 2012) targeting 130-138 bp (including primers) of the mitochondrial 16S rRNA locus, 115 and a pan-vertebrate primer set (12SV5F, 12SV5F; Riaz et al. 2011) targeting 140-143 bp (including 
primers) of the mitochondrial $12 \mathrm{~S}$ locus. Reactions also included human blocking primers

117 (16Smam_blkhum3; Boessenkool et al. 2012; 12S_V5_blkhum; Calvignac-Spencer et al. 2013b) and Sus blocking primers (16Smam_blkpig, 12S_V5_blkpig; Calvignac-Spencer et al. 2013b) to decrease competition from contaminating DNA from pork bait. Primers also included a 5' addition of a $33 \mathrm{bp}$ Illumina-specific sequence for addition of adapters in a second round of PCR. A minimum of two PCR replicates per sample were performed in $10 \mu \mathrm{l}$ volumes. For $16 \mathrm{~S}$, reactions included $0.2 \mu \mathrm{M}$ of each

122 primer, $1 \mu \mathrm{M}$ of human blocking primer (5x) $4 \mu \mathrm{M}$ of Sus blocking primer (20X), $200 \mu \mathrm{M}$ dNTP, $4 \mathrm{mM}$ $123 \mathrm{MgCl} 2,1 \mathrm{X}$ PCR buffer, $1.25 \mathrm{U}$ Platinum ${ }^{\circledR}$ Taq polymerase (Invitrogen), and $3 \mu$ l of template DNA. For $12412 \mathrm{~S}$, volumes were the same, except $\mathrm{MgCl}_{2}$ was reduced to $2.5 \mathrm{mM}$. Cycling conditions were $10 \mathrm{~min}$ at $95^{\circ} \mathrm{C}$, followed by 42 cycles $(16 \mathrm{~S})$ or 47 cycles $(12 \mathrm{~S})$ of $30 \mathrm{~s}$ at $95^{\circ} \mathrm{C}, 30 \mathrm{~s}$ at $64^{\circ} \mathrm{C}$, and $1 \mathrm{~min}$ at $72{ }^{\circ} \mathrm{C}$, with a final extension of $10 \mathrm{~min}$ at $72{ }^{\circ} \mathrm{C}$. Following the initial PCR, sample replicates were pooled, and a

127 second PCR was conducted to add Illumina flow cell binding sequences and unique $8 \mathrm{bp}$ sample specific indexes to each end of each amplicon. Reactions included $1 \mu$ l each of the forward and reverse Illumina index tags, 2.5 ul of 10X Qtaq buffer, 1.5 Mm MgCl2, and 1.25 units of Qiagen Qtaq. Cycling conditions were $3 \mathrm{~min}$ at $94^{\circ} \mathrm{C}$, followed by 6 cycles of $45 \mathrm{~s}$ at $94{ }^{\circ} \mathrm{C}, 60 \mathrm{~s}$ at $50^{\circ} \mathrm{C}$, and $1 \mathrm{~min}$ at $72{ }^{\circ} \mathrm{C}$, with a final extension of $10 \mathrm{~min}$ at $72{ }^{\circ} \mathrm{C}$. All PCR reactions were prepared in a UV-sterilized laminar flow hood, and 132 included no-template negative controls to test for contamination. None of the extraction negative 133 controls and no-template PCR negative controls showed any sign of amplification when run on an 134 agarose gel, so they were not included in sequencing libraries. Samples were purified and normalized using SequalPrep ${ }^{\text {TM }}$ Normalization Plates (Thermo-Fisher Scientific; Waltham, MA). All samples were 136 pooled in equimolar concentration, and pools were concentrated using standard Ampure XP beads 137 (Beckman Coulter; Indianapolis, IN). The concentrated pool was quantified using a Qubit fluorometer 138 and quality checked using a Bioanalyzer High Sensitivity Kit. The library was sequenced on an Illumina 139 MiSeq as $30 \%$ of a 500 cycle 2 x 250 Paired-end sequencing run. 


\section{Sequence filtering}

141 After demultiplexing, paired ends were merged for each sample using PEAR v. 0.9.6 (Zhang et al.

142 2014) with default parameters, except minimum overlap $(-v)=100$, minimum length $(-n)=100$, and

143 quality score threshold $(-q)=15$. We then separated $12 S$ and $16 S$ sequences and removed primers and

144 remaining adapters with cutadapt v. 1.3 (Martin 2011), keeping sequences with a minimum length (-m)

145 of 10 . Sequences had been previously trimmed to minimum 100 . Mean \pm 1 s.e. length after trimming

146 was $109.78 \pm 0.13$ for $12 \mathrm{~S}$ and $93.70 \pm 0.08$ for $16 \mathrm{~s}$. Sequences were subsequently pooled and filtered

147 for chimeras with the identify_chimeric_seqs.py script and the usearch61 method within the QIIME

148 environment (Caporaso et al. 2010; Edgar 2010). We used the pick_de_novo_otus.py script with the

149 uclust method to cluster sequences into OTUs (Operational Taxonomic Units) at 97\% similarity, create a

150 table of OTU frequency in each sample, and pick representative sequences (Caporaso et al. 2010; Edgar

151 2010). We removed OTUs with fewer than 20 sequences using the filter_otus_from_otu_table.py script

152 (Caporaso et al. 2010). A threshold of 20 sequence reads was chosen because all OTUs with fewer than

15320 reads had no similarity to any vertebrate species and were thus likely artefactual. To remove OTUs

154 from likely contaminants such as the pork bait, human DNA, and bacteria, we conducted an initial BLAST

155 search and removed all OTUs with a top hit for Sus, Homo, or bacteria. For 12S, we used this initial

156 BLAST search to separate OTUs into mammal, bird and reptile groups for downstream taxonomic

157 assignment.

158 Taxonomic assignment

159 For mammalian taxonomic assignment, we compared two methods: the most commonly used

160 method BLAST (Altschul et al. 1990) and the new probabilistic taxonomic placement method PROTAX

161 (Somervuo et al. 2017). For non-mammals, only BLAST was used. BLAST searches were conducted

162 against the National Center for Biotechnology Information (NCBI) non-redundant database with default 
settings except for e-value $\leq 1 \mathrm{e}-6$, and percent identity $\geq 95 \%$. We processed the BLAST output in two ways for each OTU: selecting the top hit, and by using the lowest common ancestor (LCA) algorithm in MEGAN 5.11.3 (Huson et al. 2007; default settings except top percent = 5).

For PROTAX, we generated probabilities of taxonomic placement for each OTU at four taxonomic ranks (order, family, genus, and species) (Somervuo et al. 2016). PROTAX is a statistical wrapper that processes the output of one or more other taxonomic assignment methods and takes into account the uncertainty of taxonomic assignment contributed by species that do not have reference sequences. Briefly, this is achieved by training a model against a reference dataset that includes all available reference sequences for the taxon of interest, plus a full Linnaean taxonomy for that taxon which includes all named species, including those that do not have reference sequences. By taking into account the taxonomic information content of both the gene sequence being assigned and the reference sequence database, PROTAX removes the inherent 'over-assignment' bias of other taxonomic assignment software. This makes PROTAX especially suited for markers that have highly incomplete reference databases, such as $16 \mathrm{~S}$ and $12 \mathrm{~S}$.

For the reference sequence databases, we downloaded all available mammalian mitochondrial 16S and $12 S$ sequences from GenBank, randomly truncated each species to a maximum of 10 sequences via a Fisher-Yates shuffle, removed ambiguous bases, and used ecoPCR within OBITools 1.2.6 (Boyer et al. 2016; Ficetola et al. 2010) to extract the amplicon regions used in this study (Clark et al. 2016). This resulted in 5243 16S sequences representing 1733 species and 5395 12S sequences representing 1935 species. For the taxonomic database, we downloaded full ranks for the 6711 species in the NCBI Mammalia taxonomy. We are aware that the NCBI taxonomy is not complete for the Mammalia, but the important benefit is that the names are consistent between the sequence and taxonomic databases, and even this incomplete Mammalia taxonomy has ca. 3.8X and 3.4X the number of species as 
represented in the $16 \mathrm{~S}$ and $12 \mathrm{~S}$ reference-sequence databases, respectively, which allows us to contrast

187 PROTAX with BLAST.

To start, all OTU representative sequences were pairwise compared to the reference sequences using

LAST version 744 (Kielbasa et al. 2011), and the PROTAX model used the maximum and second-best

similarities. We parameterized three PROTAX models, one unweighted model in which all mammal

species were given an equal prior weight of being in the sampling location, and two weighted models in

which species independently known to be present in Panama (Panama-weighted) or $\mathrm{BCl}$ ( $\mathrm{BCl}$-weighted)

prior probability of $10 \%$. The accuracy and bias of the trained PROTAX model at each taxonomic rank

were estimated by plotting the cumulative predicted probabilities against the cumulative number of

cases in which the outcome with the highest probability was correct when training the model.

For all methods (BLAST top hit, BLAST plus MEGAN, and PROTAX unweighted and weighted), we

$\mathrm{BCl}$ (rate of correct assignment) and the percentage of OTUs assigned to a genus or species known to

directly test the probability that an OTU was assigned incorrectly to a species known from $\mathrm{BCl}$. However,

204 rank.

\section{Transect counts and camera trapping}

206 We compared results from fly metabarcoding with results of two traditional survey methods. First,

207 diurnal mammal transect counts were carried out yearly from 2008-2015 during January and/or

208 February of each year. Transects covered all trails on $\mathrm{BCl}$ (Fig 1) each year, with a mean distance of 120 
$209 \mathrm{~km}$ walked per year. An average walking rate of $1 \mathrm{~km} / \mathrm{hr}$ was maintained. Each mammal sighting

210 recorded date, time, location, species, and number of individuals. All censuses were conducted between

$21106: 40$ and 12:00 and were thus unlikely to detect nocturnal animals. In 2015, the year fly sampling was

212 conducted, $151.1 \mathrm{~km}$ was surveyed from 24 Jan to 22 Feb. Transect counts started XXX days before and

213 overlapped with fly and camera trap sampling.

214 Second, camera traps were operated continuously across BCl from 2008-2015. A mean of 25 (range

215 19-34) trail cameras (PC900 and RC55 Reconyx Inc., Holmen, Wisconsin) were mounted at knee height,

216 with a spacing of 500-1000 meters. Thus, they were unlikely to capture arboreal or volant species.

217 Additional cameras were deployed for shorter periods. Cameras were checked at least once every 6-7

218 months and replaced or repaired if no longer functioning. This effort resulted in a total of 39,151 total

219 camera-days. For the period concurrent with fly collection, Feb 10 to May 5 2015, 26 cameras were

220 active for the entire period, resulting in a total effort of 1,967 camera-days.

\section{Methods comparison}

222 We compared the total number of mammal species detected by fly metabarcoding with the total

223 number of species detected by concurrent (2015) and long term (2008-2015) transect counts and

224 camera trapping. In addition, we fit species accumulation curves for each of the 2015 surveys using the

225 specaccum function in the R package vegan 2.4-1 (Oksanen et al. 2016) with method= random and

22610,000 permutations.

\section{Results}

\section{Transect counts and camera trapping}


In the 2015 transect campaign, we detected 13 mammal species, and in the 2008-2015 transect campaign, we detected 17 mammal species. In the concurrent 2015 camera trapping campaign, we detected 17 mammal species, and in the 2008-2015 camera trapping campaign, we detected 26 mammal species. The species detected by the two methods overlapped only partially, so that the 2015 datasets combined detected a total of 22 mammal species, and the 2008-2015 datasets combined detected a total of 30 mammal species (Table X).

\section{Fly metabarcoding}

Fly collection, extraction and sequencing.

237 A total of 1084 flies were collected and pooled into 102 pooled samples and 24 single-fly samples. We obtained 2,780,574 initial reads, which were reduced to $2,288,009$ after quality filtering $(1,504,44016 \mathrm{~S}$; 783,569 12S). OTU clustering, removal of OTUs with fewer than 20 reads, and removal of OTUs assigned to human, Sus (pork bait), and bacteria resulted in a final set of 54 OTUs for $16 \mathrm{~S}$ and 63 OTUs for $12 \mathrm{~S}$ (49 mammal, 12 bird, and 2 reptile OTUs; OTU sequences in supplementary file S1). After quality filtering

242 and removal of human, Sus, and bacterial reads, the mean number of reads per OTU was 8401 (SD = $24346578)$ for $16 S$ and $4585(S D=13029)$ for $12 S$, and the mean number of reads per sample was 2525 (SD $244=3098)$ for $16 \mathrm{~S}$ and $2218(\mathrm{SD}=4582)$ for $12 \mathrm{~S}$ for pooled samples. For single-fly samples, the mean 245 number of reads per sample was $3744(S D=3195)$ for $16 S$ and $87(S D=422)$ for $12 S$.

\section{Mammal species detection}

247 The number of mammal species detected differed between assignment methods (Fig 2a). BCl-

248 weighted PROTAX resulted in the detection of 20 total mammal species (Table 1), the most of any 249 method (16 species from 53 OTUs with 16S, 13 species from 37 OTUs with 12S, and 9 species with both 250 markers). Assignment of multiple OTUs to the same species is expected in metabarcoding since the 
sequence-clustering step typically applies a single similarity threshold across all OTUs, which can result

252 in species splitting. Fifteen of these 20 species had PROTAX probabilities of $>0.9$, but several had

253 somewhat low PROTAX probabilities despite their known presence on BCI (Table 1). Panama-weighted

254 and unweighted PROTAX resulted in detection of 19 total mammal species (13 with $16 \mathrm{~S}$ and 12 with

255 12S).

BLAST top hit and BLAST plus MEGAN both resulted in detection of fewer mammal species (15 and 13

257 respectively). The percentage of OTUs assigned to a species known from $\mathrm{BCl}$ was higher for PROTAX

258 than for BLAST (Fig 2b). Rates of mammal OTUs assigned to species not present on $\mathrm{BCl}$ (clear false

259 positives) were also generally higher for BLAST than for PROTAX, but some clear false positives were still

260 present with all assignment methods, although no clear false positives at the genus level occurred with

261 BCl-weighted PROTAX (Fig 2c).

262 Effect of fly pooling

263 From the 24 flies that were extracted both singly and in pools, 4 total mammal species were detected

264 from the pooled samples, whereas 10 total mammal species were detected from the same 24 flies when

265 extracted singly. From the single fly extractions, a range of 1-4 species were detected from each fly.

Non-mammal species detection

267 The $12 \mathrm{~S}$ primers also detected several birds and one lizard. We were only able to assign one OTU to 268 the species level, wattled jacana (Jacana jacana). Two OTUs were assigned to birds at the genus level, 269 one to an antshrike species (genus Thamnophilus, likely atrinucha or doliatus; both known from $\mathrm{BCl}$ ), 270 and one to a trogon species (genus Trogon, of which 5 species are known from $\mathrm{BCl}$ ). Several OTUs were

271 assigned the family Anatidae; however, these were a $100 \%$ match to many species within that family, 272 and six species from Anatidae are known from $\mathrm{BCl}$. We also assigned one reptile OTU to the whiptail 
273 lizards (family Teiidae) which is likely either Ameiva festiva or Ameiva leptophrys, the two Teiidae

274 species known from $\mathrm{BCl}$.

\section{Methods comparison}

276

277

278

279

280

281

282

In 2015, we detected a greater number of mammal species in 29 days of carrion-fly metabarcoding than were detected by either 29 days of transect counts or 84 calendar days of camera trapping carried out concurrently (metabarcoding $=20$ species; camera trapping $=17$ species, transect counts $=13$ species, Fig 3). We detected more mammal species with fly metabarcoding in 2015 than with eight years of transect counts from 2008-2015, but fewer than with eight years of camera trapping (transect counts 2008-2015 = 17; camera trapping 2008-2015 = 26). Using all three methods combined, we detected a total of 27 mammal species in 2015, and 34 mammal species from 2008-2015. Visual inspection of the species accumulation curves from 2015 (Figure 4) suggests that additional carrion fly sampling effort, or greater sequencing depth, would likely have resulted in a greater number of species detections, whereas the transect count and camera-trap datasets were nearing asymptotes.

Of the 20 species detected with fly metabarcoding (Table 1), four were not detected by camera traps or transect counts from 2008-2015. These included Derby's woolly opossum (Caluromys derbianus), a species from the genus Canis that was a $100 \%$ match to reference sequences of domestic dog (C. domesticus) and coyote (C. latrans) and two bat species. However, metabarcoding did not detect three species commonly detected with transect counts or camera trapping: paca (Agouti paca), white-nosed coati (Nasua narica), and red brocket deer (Mazama americana) (Table 2). Moreover, the CentralAmerican agouti (Dasyprocta punctata), a large rodent that is by far the most common mammal on $\mathrm{BCl}$, was detected in just one metabarcoding sample, and only by the $12 \mathrm{~S}$ marker.

\section{Discussion}


In total, we detected a larger number of mammal species with carrion fly metabarcoding than with transect counts or camera trapping carried out during the same general time and with similar levels of 297 effort. Of the 20 species detected with fly metabarcoding (Table 1), four were not detected by camera298 traps or transect counts. Two were bat species that are unlikely to be detected by camera traps or 299 diurnal transect counts. The third, Derby's woolly opossum (Caluromys derbianus), is arboreal and 300 nocturnal, but it has been commonly observed at night feeding on canopy flowers. The fourth and most unexpected detection was a species from the genus Canis, which was a $100 \%$ match to reference 302 sequences of both domestic dog (C. domesticus) and coyote (C. latrans). Domestic dogs are not 303 permitted on $\mathrm{BCl}$, but they are present on the mainland $<1$ kilometer away at the nearest point. 304 Coyotes have never been detected on $\mathrm{BCl}$ but have been expanding in Panama (Bermudez et al. 2013) 305 and have recently been photographed by camera traps in the adjacent Soberania National Park 306 (www.teamnetwork.org). It is possible that a fly fed on a canid on the mainland, and then flew to $\mathrm{BCl}$. 307 Little is known about flight and foraging distances for carrion flies; however, Lee (2016) detected a blowfly movement distance of $3 \mathrm{~km}$ in tropical Malaysia. It is also possible that ex-situ contaminating 309 DNA from a domestic dog was introduced in the lab or was present in PCR reagents, although this seems 310 unlikely.

Although fly metabarcoding detected more mammal species than did the two more traditional 312 techniques, a clear shortcoming was complete failure to detect three abundant species that are 313 commonly detected by transect counts and camera trapping: the common rodent Agouti paca, the 314 most common carnivore Nasua narica, and a common ungulate (Mazama americana). Also, the agouti 315 (Dasyprocta punctata) was barely detected, with just one read in one marker. Metabarcoding did, 316 however, detect two other rodent species, red tailed squirrel (Sciurus granatensis), and Tome's spiny-rat 317 (Proechimys semispinosus), and two confamilial carnivores, crab-eating raccoon (Procyon cancrivorus), 318 and kinkajou (Potos flavus) (Table 1). 
There are three general explanations for why common species can fail to be detected with

321 metabarcoding. The first explanation is that reference sequences for those species are missing from the 322 reference database. Only 59\% and $62 \%$ of mammal species and $82 \%$ and $85 \%$ of genera known from $\mathrm{BCl}$ 323 are represented in our $16 \mathrm{~S}$ and $12 \mathrm{~S}$ reference databases, respectively. However, in this case, all three 324 species were present in our reference databases to varying extents: paca was represented in our 325 database by $12 \mathrm{~S}$, coati was represented by both markers, albeit only at the genus level (South American 326 Coati; Nasua nasua), and red brocket deer was represented by both markers at the species level. Given 327 that PROTAX was able to place $98 \%$ of 16 S OTUs, and $74 \%$ of 12 S OTUs to at least the genus level (Fig 2), 328 missing reference sequences are not a convincing explanation for detection failure. However, in less329 studied areas, incomplete reference databases are more likely to hamper species assignment, and thus 330 investment in building reference databases should continue.

331 The second potential explanation for why some common species were not detected with 332 metabarcoding is that there were mismatches in our $16 \mathrm{~S}$ and $12 \mathrm{~S}$ primers with binding sites for these 333 species. This could result in failed PCR amplification, even if DNA from these species was present in fly 334 samples. For paca, all nine reference sequences on GenBank have one mismatch in the forward primer, 3356 bp from the $3^{\prime}$ end. One mismatch is unlikely to completely prevent amplification but could reduce 336 PCR efficiency, especially in mixed samples with other targets. For red brocket deer, no primer 337 mismatches are observed for $12 \mathrm{~S}$. For $16 \mathrm{~S}$, the forward primer has one mismatch with all Genbank 338 reference sequences, but at the far $5^{\prime}$ end. We could not evaluate primer mismatches for white-nosed 339 coati at the species level, but both $16 \mathrm{~S}$ and $12 \mathrm{~S}$ primer sets have one $5^{\prime}$ mismatch to the congener 340 Nasua nasua. Currently available GenBank reference sequences also might not account for local 341 sequence variants with primer mismatches. 
The third, and most likely, explanation is that fly feeding preferences contribute to sampling bias.

Although carrion flies feed on carcasses of dead animals, we suspect that the main source of eDNA in our samples is feces. Primates were the most commonly detected species, with mantled howler monkey (Alouatta palliata) and Geoffroy's spider monkey (Ateles geoffroyi) identified in $98 \%$ and $85 \%$ of samples respectively. Monkeys, and particularly howler monkeys, which feed on leaves, produce an abundant quantity of soft scat that can be easily consumed by flies, while rodents such as agoutis and pacas, and ungulates such as red-brocket deer, have smaller and harder scats that may not be as attractive to flies. It is notable that some rodent species were detected nonetheless, but this could have been from carrion, and not from feces. Likewise, Calvignac-Spencer et al. (2013b) were able to detect forest ungulates with carrion flies. Further research into the source of carrion fly derived eDNA, and how this affects detection is needed. At the least, fly metabarcoding does seem to work well for primates, as all four primate species present on $\mathrm{BCl}$ were detected, and Calvignac-Spencer et al. (2013b) also detected many primates with carrion fly metabarcoding in Cote d'Ivoire and Madagascar.

For species assignment, PROTAX, especially weighted PROTAX, outperformed other assignment methods. With weighted PROTAX, we were able to assign nearly all OTUs to genus, and most to species, especially with $16 \mathrm{~S}$. For those OTUs that we could only place at the genus level, we could assign all but two to species by using prior knowledge of the vertebrate community on $\mathrm{BCl}$, since only one member of each genus is present on the island. Weighted PROTAX resulted in fewer clearly false positives (species not known from $\mathrm{BCl}$ ) than either BLAST or unweighted PROTAX (Fig 2c). However, even weighted PROTAX produced a few false positive assignments at the species level. In cases where it is important to be conservative with species assignment, and eliminate or minimize false assignments, selecting a high probability threshold (e.g. 0.95) recommended. All but one of our false positive assignments from weighted PROTAX at the species level, and all at the genus level, had PROTAX probabilities of $<0.9$ (Supplementary file S2). A high cutoff value, however, trades off minimizing false positives for more 
false negatives. That is, we detected several species known from $\mathrm{BCl}$ at assignment probabilities of $<0.9$

367 (table 1), and so with a higher probability threshold, we would have considered those species (although not those genera) as undetected even though they are present. The results from our weighted datasets suggest that an effective way to simultaneously reduce false positive (overly confident) and false negative (overly conservative) assignments in PROTAX is to use expert knowledge to assign high prior probabilities to species known to exist in the region (Fig. 2).

As evidenced by the 24 flies that we analyzed individually and in pools, pooling reduced the

373 number of species detected. When flies were extracted individually, we detected 10 species, including ocelot (Leopardus pardalis), Hoffmann's two-toed sloth (Choloepus hoffmanni), collared peccary (Pecari tajacu), kinkajou, crab-eating raccoon, red-tailed squirrel, and all 4 primates. In the 2 pooled samples from these same 24 flies, we only detected collared peccary, kinkajou, and the two most commonly detected primate species. Hoffmann's two-toed sloth was only detected in one of the single-fly extractions, and was not detected in any of the pooled samples. Thus, if we had not extracted some flies 379 singly, this species would have been missed entirely. Pooling is more likely to affect detection of rare species, as their DNA may be outcompeted during PCR by more abundant DNA of common species. It is also likely that species with more primer mismatches will be outcompeted by species with greater PCR 382 efficiency if DNA of two such species exist in the same pool. Pooling of flies, however, allows for far 383 fewer DNA extractions and individual PCRs, which substantially reduces labor and cost (up to 16 fold in 384 this example), which may allow many more flies to be processed, ultimately possibly increasing the number of species detected. If a pooling strategy is employed, and there are particular target species of 386 concern for which detection is essential, it may be desirable to include species-specific primers in 387 addition to general primers (Schubert et al. 2014). It is also possible that the high number of PCR cycles we employed led to 'PCR runaway', in which common amplicons became exponentially abundant in pooled samples at the expense of less-common amplicons. Thus, we advise future studies to optimize 
the number of PCR cycles with quantitative PCR prior to metabarcoding (Murray et al. 2015). Finally, the

391 discrepancy in species detection between pooled and single fly samples could have been the result of

392 insufficient sequencing depth. Because single fly and pooled samples were sequenced at the same

393 depth, it is possible that for pooled samples, sequencing depth was not sufficient to detect all species in

394 the sample.

The use of two different markers (12S and $16 \mathrm{~S})$ resulted in the detection of more species than either marker alone. Thus, we recommend using multiple markers to improve species detection. The inclusion

397 of the $12 \mathrm{~S}$ marker allowed us to detect more mammal species, and also allowed detection of birds and reptiles. This marker, however, appears to have relatively poor information content for discriminating birds and reptiles at the species level. Thus, if the goal is to detect non-mammalian vertebrates it may be preferable to employ additional group-specific markers. Multiple markers optimized for different groups or families could be run simultaneously, which should increase overall detection rates. For mammals, $12 \mathrm{~S}$ species assignment were generally lower confidence than with $16 \mathrm{~S}$ (supplementary file $\mathrm{S} 2$ ), and a greater proportion of $12 \mathrm{~S}$ OTUs could not be assigned at the genus or species level (Fig $2 \mathrm{~b}$ ). The standard cytochrome oxidase I (COI) barcode region is by far the most represented in reference databases, but lack of conserved sites in the coding region of COI make primer design for amplification 406 of short fragments from degraded DNA difficult for a wide range of taxa (Deagle et al. 2014). Thus, 407 mitochondrial gene regions such as $16 \mathrm{~S}$ and $12 \mathrm{~S}$ are more appropriate for metabarcoding studies 408 targeting degraded DNA. 
413 but even with the blocking primers, Sus DNA was still commonly amplified. Sus OTUs accounted for $68 \%$

414 of total $16 \mathrm{~S}$ reads and $43 \%$ of total $12 \mathrm{~S}$ reads in our dataset. In a small exploratory study (unpublished

415 data), we found that increasing Sus blocking primer concentrations to $20 x$ as opposed to the $5 x$

416 concentration used by Calvignac-Spencer et al. (2013b) led to increased detection of non-Sus DNA. If

417 pork is used as bait, we recommend taking as much care as possible to reduce contact of flies with the

418 bait to reduce contamination and to ensure enough sequencing depth so that non-Sus amplicons are

419 still sequenced even if they are in the minority. Use of other baits such as chicken or fish, or non-

420 biological commercial fly baits may mitigate these concerns, but the $12 \mathrm{~S}$ marker will still amplify chicken

421 or fish DNA, and the $16 \mathrm{~S}$ marker will also amplify fish DNA (Cannon et al. 2016). Finally, most of the fly

422 samples we collected were from a relatively clustered area (Fig 1 ) whereas transect counts and camera

423 trapping were more widely distributed throughout the island. It is possible that more widely distributed

424 fly sampling might have further improved species detection.

425 For maximal species detection, metabarcoding, camera-trapping, and transect counts could be used

426 simultaneously, as they are complementary in both information content. The three techniques

427 combined detected more species than any one alone. Conveniently, add fly collection to a visual

428 transect campaign would add little additional field time or cost. Also, if the goal was to survey

429 biodiversity of both vertebrates and invertebrates, fly traps could be paired with other types of insect

430 traps e.g. pitfall traps, and fly DNA and bulk DNA from traps could be amplified with invertebrate

431 primers and included in sequencing runs (Ji et al. 2013; Yu et al. 2012).

\section{Conclusions}

This field test confirms that carrion fly-derived DNA metabarcoding is a powerful tool for mammal

434 biodiversity surveys, already on par with other commonly used methods. A relatively small effort (29 
days of fly sampling conducted by one individual) detected the majority of the non-volant mammal

436 species resident on $\mathrm{BCl}$, and a greater number of mammal species than were detected independently by

437 camera trapping or diurnal transect counts with similar sampling effort. However, fly metabarcoding

438 failed or almost failed to detect four abundant species that are easily detected with the other methods.

439 Also, metabarcoding might not provide reliable estimates of species relative abundance (Schnell et al.

440 2015). Thus, we do not advocate the replacement of other methods with carrion fly metabarcoding.

441 Instead, we suggest that fly metabarcoding can be used to augment existing methods. This way, we can

442 make more of our expensive field time. Our results also suggest some methodological modifications that

443 will likely increase the detection power of carrion fly metabarcoding, including more complete reference

444 sequence databases, optimization of PCR conditions, multiple custom markers, greater sampling effort,

445 and greater sequencing depth. Individual fly metabarcoding could also increase detection power, but at

446 a larger cost in the lab. As lab costs drop further and as reference databases of complete mitochondrial

447 genomes becomes readily available (Tang et al. 2014), this method promises to help us achieve more

448 rapid characterization and monitoring of vertebrate communities.

\section{Acknowledgments}

450 This work was funded by a Smithsonian Tropical Research Institute fellowship (TR). Camera trapping and

451 transect counts was funded by the STRI Terrestrial Environmental Studies Project, the U.S. Department

452 of Education Math-Science Partnerships Project (JG), and private funds from Gregory E. Willis. We also

453 thank Gregory E. Willis for years of mammals census field work. DY was supported by the National

454 Natural Science Foundation of China (31400470, 41661144002, 31670536, 31500305, GYHZ1754), the

455 Ministry of Science and Technology of China (2012FY110800), the University of East Anglia, and the

456 State Key Laboratory of Genetic Resources and Evolution at the Kunming Institute of Zoology (GREKF13-

457 13, GREKF14-13, GREKF16-09). 


\section{References}

459

460

461

462

463

464

465

466

467

468

469

470

471

472

473

474

475

476

477

478

479

480

481

482

483

484

485

486

487

488

489

490

491

492

493

494

495

496

497

498

499

500

501

Altschul, S. F., Gish, W., Miller, W., Myers, E. W., \& Lipman, D. J. (1990). BASIC LOCAL ALIGNMENT SEARCH TOOL. [Article]. Journal of Molecular Biology, 215(3), 403-410. doi: 10.1006/jmbi.1990.9999

Andersen, K., Bird, K. L., Rasmussen, M., Haile, J., Breuning-Madsen, H., Kjaer, K. H., . . . Willerslev, E. (2012). Meta-barcoding of 'dirt' DNA from soil reflects vertebrate biodiversity. Mol Ecol, 21(8), 1966-1979. doi: 10.1111/j.1365-294X.2011.05261.x

Beaudrot, L., Ahumada, J. A., O'Brien, T., Alvarez-Loayza, P., Boekee, K., Campos-Arceiz, A., . . . Andelman, S. J. (2016). Standardized Assessment of Biodiversity Trends in Tropical Forest Protected Areas: The End Is Not in Sight. PLoS Biol, 14(1), e1002357. doi: 10.1371/journal.pbio.1002357

Bermudez, C. S. E., Gonzalez, D. D., \& Garcia, S. G. (2013). Ticks (Acari: Ixodidae, Argasidae) of Coyotes in Panama. [Article]. Systematic and Applied Acarology, 18(2), 112-115.

Boessenkool, S., Epp, L. S., Haile, J., Bellemain, E., Edwards, M., Coissac, E., . . Brochmann, C. (2012). Blocking human contaminant DNA during PCR allows amplification of rare mammal species from sedimentary ancient DNA. Mol Ecol, 21(8), 1806-1815. doi: 10.1111/j.1365-294X.2011.05306.x

Bohmann, K., Evans, A., Gilbert, M. T. P., Carvalho, G. R., Creer, S., Knapp, M., . . de Bruyn, M. (2014). Environmental DNA for wildlife biology and biodiversity monitoring. [Review]. Trends Ecol Evol, 29(6), 358-367. doi: 10.1016/j.tree.2014.04.003

Bohmann, K., Schnell, I. B., \& Gilbert, M. T. P. (2013). When bugs reveal biodiversity. [News Item]. Mol Ecol, 22(4), 909-911. doi: 10.1111/mec.12221

Boyer, F., Mercier, C., Bonin, A., Le Bras, Y., Taberlet, P., \& Coissac, E. (2016). obitools: a unix-inspired software package for DNA metabarcoding. Mol Ecol Resour, 16(1), 176-182. doi: 10.1111/17550998.12428

Calvignac-Spencer, S., Leendertz, F. H., Gilbert, M. T. P., \& Schubert, G. (2013a). An invertebrate stomach's view on vertebrate ecology Certain invertebrates could be used as "vertebrate samplers" and deliver DNA-based information on many aspects of vertebrate ecology. Bioessays, 35(11), 1004-1013. doi: 10.1002/bies.201300060

Calvignac-Spencer, S., Merkel, K., Kutzner, N., Kuhl, H., Boesch, C., Kappeler, P. M., . . Leendertz, F. H. (2013b). Carrion fly-derived DNA as a tool for comprehensive and cost-effective assessment of mammalian biodiversity. Mol Ecol, 22(4), 915-924. doi: 10.1111/mec.12183

Cannon, M. V., Hester, J., Shalkhauser, A., Chan, E. R., Logue, K., Small, S. T., \& Serre, D. (2016). In silico assessment of primers for eDNA studies using PrimerTree and application to characterize the biodiversity surrounding the Cuyahoga River. Scientific Reports, 6. doi: 10.1038/srep22908

Caporaso, J. G., Kuczynski, J., Stombaugh, J., Bittinger, K., Bushman, F. D., Costello, E. K., . . . Knight, R. (2010). QIIME allows analysis of high-throughput community sequencing data. Nature methods, 7(5), 335-336. doi: 10.1038/nmeth.f.303

Clark, K., Karsch-Mizrachi, I., Lipman, D. J., Ostell, J., \& Sayers, E. W. (2016). GenBank. [Article]. Nucleic Acids Research, 44(D1), D67-D72. doi: 10.1093/nar/gkv1276

Deagle, B. E., Jarman, S. N., Coissac, E., Pompanon, F., \& Taberlet, P. (2014). DNA metabarcoding and the cytochrome c oxidase subunit I marker: not a perfect match. [Article]. Biology Letters, 10(9). doi: 10.1098/rsbl.2014.0562

Edgar, R. C. (2010). Search and clustering orders of magnitude faster than BLAST. Bioinformatics, 26(19), 2460-2461. 
Ficetola, G. F., Coissac, E., Zundel, S., Riaz, T., Shehzad, W., Bessière, J., . . Pompanon, F. (2010). An In silico approach for the evaluation of DNA barcodes. [journal article]. Bmc Genomics, 11(1), 434. doi: 10.1186/1471-2164-11-434

Gillett, C. P. D. T., Johnson, A. J., Barr, I., \& Hulcr, J. (2016). Metagenomic sequencing of dung beetle intestinal contents directly detects and identifies mammalian fauna. bioRxiv. doi: $10.1101 / 074849$

Glanz, W. E. (1982). The terrestrial mammal fauna of Barro Colorado Island: censuses and long-term changes. In E. G. Leigh, A. S. Rand \& D. M. Windsor (Eds.), The Ecology of a Tropical Forest: Seasonal Rhythms and Long-Term Changes (pp. 455-468). Washington DC: Smithsonian Institution Press.

Huson, D. H., Auch, A. F., Qi, J., \& Schuster, S. C. (2007). MEGAN analysis of metagenomic data. Genome Research, 17(3), 377-386. doi: 10.1101/gr.5969107

Ji, Y., Ashton, L., Pedley, S. M., Edwards, D. P., Tang, Y., Nakamura, A., . . Yu, D. W. (2013). Reliable, verifiable and efficient monitoring of biodiversity via metabarcoding. Ecology letters, 16(10), 1245-1257. doi: 10.1111/ele.12162

Kielbasa, S. M., Wan, R., Sato, K., Horton, P., \& Frith, M. C. (2011). Adaptive seeds tame genomic sequence comparison. [Article]. Genome Research, 21(3), 487-493. doi: 10.1101/gr.113985.110

Lee, P. S. (2016). A blowfly-derived dna approach to assess diversity of tropical mammals. PhD Thesis, University of Malaysia

http://studentsrepo.um.edu.my/6715/.

Lee, P. S., Gan, H. M., Clements, G. R., \& Wilson, J.-J. (2016). Field calibration of blowfly-derived DNA against traditional methods for assessing mammal diversity in tropical forests. Genome, 59(11), 1008-1022. doi: 10.1139/gen-2015-0193

Lee, P. S., Sing, K. W., \& Wilson, J. J. (2015). Reading Mammal Diversity from Flies: The Persistence Period of Amplifiable Mammal mtDNA in Blowfly Guts (Chrysomya megacephala) and a New DNA Mini-Barcode Target. PLoS One, 10(4). doi: 10.1371/journal.pone.0123871

Logue, K., Keven, J. B., Cannon, M. V., Reimer, L., Siba, P., Walker, E. D., . . Serre, D. (2016). Unbiased Characterization of Anopheles Mosquito Blood Meals by Targeted High-Throughput Sequencing. PLOS Neglected Tropical Diseases, 10(3), e0004512. doi: 10.1371/journal.pntd.0004512

Martin, M. (2011). Cutadapt removes adapter sequences from high-throughput sequencing reads. EMBnet.journal; Vol 17, No 1: Next Generation Sequencing Data Analysis.

Murray, D. C., Coghlan, M. L., \& Bunce, M. (2015). From Benchtop to Desktop: Important Considerations when Designing Amplicon Sequencing Workflows. PLoS One, 10(4), e0124671. doi: 10.1371/journal.pone.0124671

Nichols, R. V., Konigsson, H., Danell, K., \& Spong, G. (2012). Browsed twig environmental DNA: diagnostic PCR to identify ungulate species. [Article]. Mol Ecol Resour, 12(6), 983-989. doi: 10.1111/j.17550998.2012.03172.x

Oksanen, J., Blanchet, F. G., Friendly, M., Kindt, R., Legendre, P., McGlinn, D., . . Wagner, H. (2016). vegan: Community Ecology Package. $R$ package version 2.4-1.

Riaz, T., Shehzad, W., Viari, A., Pompanon, F., Taberlet, P., \& Coissac, E. (2011). ecoPrimers: inference of new DNA barcode markers from whole genome sequence analysis. Nucleic Acids Research, 39(21), e145-e145. doi: 10.1093/nar/gkr732

Rodgers, T. W., \& Mock, K. E. (2015). Drinking water as a source of environmental DNA for the detection of terrestrial wildlife species. Conservation Genetics Resources, 7(3), 693-696. doi: 10.1007/s12686-015-0478-7

Schnell, I. B., Sollmann, R., Calvignac-Spencer, S., Siddall, M. E., Yu, D. W., Wilting, A., \& Gilbert, M. T. P. (2015). iDNA from terrestrial haematophagous leeches as a wildlife surveying and monitoring 
tool - prospects, pitfalls and avenues to be developed. Frontiers in Zoology, 12. doi: 10.1186/s12983-015-0115-z

Schubert, G., Stockhausen, M., Hoffmann, C., Merkel, K., Vigilant, L., Leendertz, F. H., \& CalvignacSpencer, S. (2014). Targeted detection of mammalian species using carrion fly-derived DNA. Mol Ecol Resour, 15(2), 285-294. doi: 10.1111/1755-0998.12306

Somervuo, P., Koskela, S., Pennanen, J., Henrik Nilsson, R., \& Ovaskainen, O. (2016). Unbiased probabilistic taxonomic classification for DNA barcoding. Bioinformatics, 32(19), 2920-2927. doi: 10.1093/bioinformatics/btw346

Somervuo, P., Yu, D. W., Xu, C., Ji, Y., Hultman, J., Wirta, H., \& Ovaskainen, O. (2017). Quantifying uncertainty of taxonomic placement in DNA barcoding and metabarcoding. Methods in Ecology and Evolution, accpeted. doi: 10.1101/070573

Taberlet, P., Coissac, E., Hajibabaei, M., \& Rieseberg, L. H. (2012). Environmental DNA. Mol Ecol, 21(8), 1789-1793. doi: 10.1111/j.1365-294X.2012.05542.x

Tang, M., Tan, M., Meng, G., Yang, S., Su, X., \& Liu, S. (2014). Multiplex sequencing of pooled mitochondrial genomes - a crucial step toward biodiversity analysis using mito-metagenomics. Nucleic Acids Res, 42. doi: 10.1093/nar/gku917

Thomsen, P. F., Kielgast, J., Iversen, L. L., Wiuf, C., Rasmussen, M., Gilbert, M. T. P., . . Willerslev, E. (2012). Monitoring endangered freshwater biodiversity using environmental DNA. Mol Ecol, 21(11), 2565-2573. doi: 10.1111/j.1365-294X.2011.05418.x

Wheat, R. E., Allen, J. M., Miller, S. D. L., Wilmers, C. C., \& Levi, T. (2016). Environmental DNA from Residual Saliva for Efficient Noninvasive Genetic Monitoring of Brown Bears (Ursus arctos). PLoS One, 11(11), e0165259. doi: 10.1371/journal.pone.0165259

Yu, D. W., Ji, Y., Emerson, B. C., Wang, X., Ye, C., Yang, C., \& Ding, Z. (2012). Biodiversity soup: metabarcoding of arthropods for rapid biodiversity assessment and biomonitoring. Methods in Ecology and Evolution, 3(4), 613-623. doi: 10.1111/j.2041-210X.2012.00198.x

Zhang, J., Kobert, K., Flouri, T., \& Stamatakis, A. (2014). PEAR: a fast and accurate Illumina Paired-End reAd mergeR. Bioinformatics, 30(5), 614-620. doi: 10.1093/bioinformatics/btt593

\section{Figure and table captions}

Figure 1 Location of carrion-fly trapping on Barro Colorado Island, Panama.

Figure 2 Comparison of taxonomic placement methods for assigning mammal OTUs to species from metabarcoding of carrion flies collected on Barro Colorado Island (BCI). A) Number of mammal species detected from $\mathrm{BCl}$ using each taxonomic method. B) Percentages of OTUs assigned to genera or species known to occur on $\mathrm{BCl}$. C) Numbers of OTUs assigned to genera or species known not to exist on $\mathrm{BCl}$ (clear false positives). 
Figure 3 Number of mammal species detected by alternative sampling methods on Barro Colorado Island, Panama.

Figure 4 Species accumulation curves for three different survey methods used to sample mammal diversity on Barro Colorado Island, Panama in 2015.

Table 1 Twenty mammal species detected from metabarcoding of carrion flies on Barro Colorado Island Panama in 2015, along with PROTAX (BCl-weighted) estimated probabilities of correct assignment at genus and species rank, and the percentage of fly pool samples that each species was detected from.

Table 2 Mammal species detected by carrion fly metabarcoding, camera trapping, and diurnal transect counts on Barro Colorado Island, Panama. For metabarcoding, values represent number of samples in which a species was detected. For camera trapping and transect counts, values are number of individuals detected.

\section{Supplementary files}

S1 FASTA file of OTU sequences.

S2 Spreadsheet of weighted and unweighted PROTAX probabilities at the Class, Family, Genus, and Species level for all OTUs.

\section{Data Accessibility}

Raw sequence data are available on the NCBI Sequence Read Archive under accession number PRJNA382243.OTU sequences are available in the supplementary information. Scripts for the PROTAX analysis will be made available on Dryad prior to publication.

\section{Author contributions}


Torrey Rodgers designed the study, carried out all fly collection field work and lab work, coordinated the data analysis, and wrote the first draft. Charles Xu performed the PROTAX analysis, Jacalyn Giacalone collected the camera trap and transect count data, Karen Kapheim conducted the sequence filtering and OTU generation bioinformatics work, Kristin Saltonstall helped with design and implementation of lab work, Marta Vargas helped with lab work management and conducted MiSeq sequencing, Douglas Yu supervised the PROTAX analysis, Panu Somervuo provided scripts and helped with the PROTAX analysis, and Patrick A. Jansen helped with field study design. W. Owen McMillan and Patrick Jansen jointly advised this work. All authors contributed to manuscript editing. 